\title{
Covalent linking peptide to hydrothermally reduced graphene oxide for ultrasensitive detection of matrix metalloproteinase 9
}

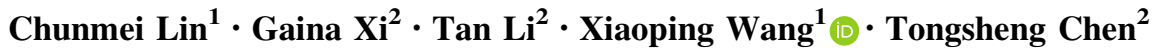

Received: 9 September 2017 / Accepted: 4 October 2017/Published online: 10 October 2017

(c) The Author(s) 2017. This article is an open access publication

\begin{abstract}
Exact assay of matrix metalloproteinase 9 (MMP9) has attracted considerable attentions for the clinical diagnosis of disease in early stage. In this report, we covalently engineer a fluorescein isothiocyanate-labeled peptide (Pep-FITC) linker containing the specific cleavage substrate of MMP9 onto the surface of hydrothermally reduced nano-graphene oxide (nrGO) to develop a FRETbased nrGO-Pep-FITC nanoprobe for ultrasensitive detection of MMP9. Upon cleavage of the Pep-FITC at the amide bond between Ser and Leu by MMP9, FITC was separated from nrGO, and fluorescence recovery of FITC was proportional to the MMP9 concentration within $0.01-0.06 \mathrm{nM}$ and $0.06-0.15 \mathrm{nM}$ ranges, respectively, in an aqueous solution, exhibiting $0.83 \mathrm{pM}$ of detection limit. This nanoprobe is stable under borate buffer $(\mathrm{pH}=7.4)$ with bovine serum albumin or $0.1 \%$ surfactant Triton 100 or Tween 20, and has good specificity for MMP9 detection, which is meaningful for MMP9-related clinical and bioanalytical applications.
\end{abstract}

Chunmei Lin and Gaina Xi contributed equally to this work.

Electronic supplementary material The online version of this article (doi:10.1007/s13204-017-0613-8) contains supplementary material, which is available to authorized users.

Xiaoping Wang

txp2938@jnu.edu.cn

1 Department of Pain Management, The First Affiliated Hospital of Jinan University, Guangzhou 510632, People's Republic of China

2 MOE Key Laboratory of Laser Life Science \& College of Biophotonics, South China Normal University, Guangzhou 510631, People's Republic of China
Keywords Reduced nano-graphene oxide · Nanoprobe · MMP9 - Ultrasensitive detection

\section{Introduction}

Matrix metalloproteinases (MMPs) are a group of proteinases that selectively degrade components of extracellular matrix (ECM) (Celentano and Frishman 1997). Of MMPs, MMP9 is implicated in various pathological conditions including diabetes (Giebel et al. 2005), hypertension (Berk et al. 2007), and cancers (Martin and Matrisian 2007). High level of MMP9 has been detected in various body fluids taken from humans with various tumors (Fernández et al. 2005; Hurst et al. 2007; Eissa et al. 2007; Mroczko et al. 2008; Shpitzer et al. 2009). MMP9 not only promotes tumor angiogenesis ( $\mathrm{Yu}$ and Stamenkovic 2000; Riedel et al. 2000) but also regulates cell adhesion by the hydrolysis of ECM resulting in tumor invasion and metastasis (Sternlicht and Werb 2001). MMP9 can directly degrade elastin, the main component of arteries, resulting in arterial stiffness and hypertension (Berk et al. 2007), and breaks down occludin, a structural element in the blood-retinal barrier, to enhance vascular permeability and diabetic retinopathy (Giebel et al. 2005). MMP9 can also digest type IV collagen, fibronectin, and laminin, and thus lead to the destruction of blood-brain barrier and brain damage (Fujimura et al. 1999; Rosenberg et al. 1998). In addition, MMP9 was reported to be involved in a series of degeneration phenomena, such as osteoarthritis and rheumatoid arthritis (Burrage et al. 2006). Therefore, accurate and sensitive detection of MMP9 has great significance for clinical diagnosis and therapy of disease in early stage.

Fluorescence resonance energy transfer (FRET)-based nanoprobes have been widely developed to detect MMPs 
in vivo (Akers et al. 2012) and in vitro ( $\mathrm{Li}$ et al. 2015). Based on the specific cleavage site of MMP2 between the Leu and Gly of Pro-Leu-Gly-Val-Arg, some nanoprobes via engineering a dye-labeled peptide (Pep-dye) containing the MMP2-specific cleavage substrate onto the surface of nanoparticle have been fabricated for sensitive MMP2 detection (Wang et al. 2014; Li et al. 2011; 2014; Feng et al. 2011; Song et al. 2013; Xi et al. 2016). Wang and coworkers (2014) used poly(m-phenylenediamine) (PMPD) as an acceptor to develop PMPD-Pep-FITC probe showing $32 \mathrm{pM}$ of detection limit for MMP2 (Wang et al. 2014). Using the good optical absorptivity of graphene oxide (GO), Song and coworkers developed a GO-PepFITC probe that could detect MMP2 at concentration as low as 35 pM (Song et al. 2013). Considering the higher optical absorptivity of reduced graphene oxide (rGO) over GO, we recently developed a non-covalent rGO-based FRET nanoprobe (nrGO/Pep-FITC) with $3 \mathrm{pM}$ of detection limit for MMP2 detection (Xi et al. 2016). Similarly, Nguyen and coworkers noncovalently bound fluorescent gold nanocluster-labeled peptide containing the specific cleavage site of MMP9 to GO for MMP9 detection, and the detection limit of this probe was $0.15 \mathrm{nM}$ (Nguyen et al. 2017). However, extremely low concentration of MMP9 in healthy human plasma, about tenfold lower than that of MMP2 (Kuyvenhoven et al. 2004), makes MMP9 detection very difficult.

In this study, we developed a covalent nrGO-based FRET nanoprobe (nrGO-Pep-FITC) for ultrasensitive MMP9 assay. As illustrated in Fig. 1, nano-GO (nGO) was firstly prepared by sonication of GO, and then carboxylated through the carboxylation reaction between $\mathrm{nGO}, \mathrm{NaOH}$, and $\mathrm{ClCH}_{2} \mathrm{COOH}$ for $3 \mathrm{~h}$ to obtain the carboxylated $\mathrm{nGO}$ (c-nGO), and the c-nGO was hydrothermally bathed under $90{ }^{\circ} \mathrm{C}$ for $1.5 \mathrm{~h}$ to get the reduced $\mathrm{nGO}$ (nrGO). Finally, the Pep-FITC $\quad\left(\mathrm{NH}_{2}\right.$-Lys-Gly-Pro-Arg-Ser-Leu-Ser-Gly-
Lys-FITC) containing the cleavage site of MMP9 between Ser and Leu (Fudala et al. 2014) was covalently conjugated with nrGO under the participation of EDC to obtain the nrGO-Pep-FITC. In the presence of MMP9, the cleavage of Pep-FITC leads to the separation of FITC from nrGO and subsequent fluorescence recovery. Moreover, the nrGOPep-FITC nanoprobe developed here is very stable under borate buffer $(\mathrm{pH}=7.4)$ with BSA or $0.1 \%$ surfactant Triton 100 or Tween 20, and exhibits 0.83 pM MMP9 detection limit.

\section{Materials and methods}

\section{Chemicals and materials}

Pep-FITC $\quad\left(\mathrm{NH}_{2}\right.$-Lys-Gly-Pro-Arg-Ser-Leu-Ser-GlyLys-FITC) was synthesized by Wuhan Fine Peptide Co. Ltd. Graphene oxide (GO) flake, latent proMMP9, 1-ethyl3-(dimethylaminopropyl)carbodiimide hydrochloride (EDC), 4-aminophenylmercuric acetate (APMA), dimethyl sulfoxide (DMSO), other reagents employed, and all buffers have been described in our previous publication (Xi et al. 2016).

\section{Synthesis of c-nGO and nrGO}

GO was dissolved in tri-distilled water and sonicated just as described in our previous publication (Xi et al. 2016). $\mathrm{NaOH}$ and $\mathrm{ClCH}_{2} \mathrm{COOH}$ were added to nGO suspension and then sonicated for $1 \mathrm{~h}$. The reaction liquid was divided into four parts, and then stirred for $0,1,2$, and $3 \mathrm{~h}$, respectively. The resultant solution was purified by repeated rinsing with water until c-nGO can be well dispersed in water (Sun et al. 2008). c-nGO solution was bathed at $90{ }^{\circ} \mathrm{C}$ for $1.5 \mathrm{~h}$ to get nrGO.

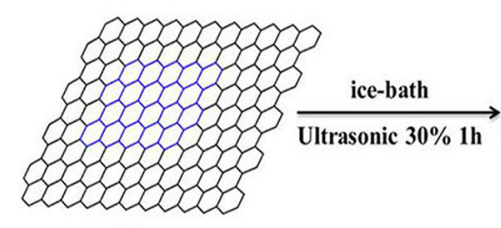

GO

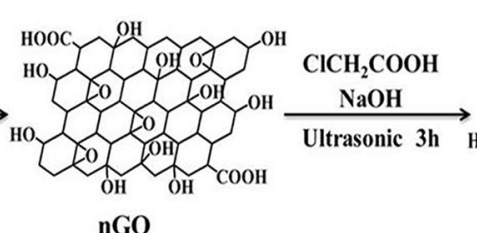

nGO

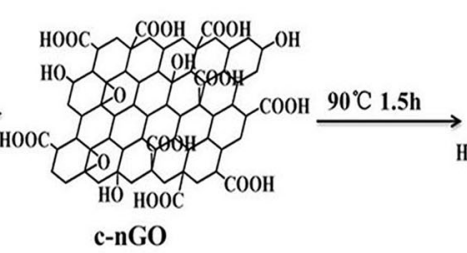

c-nGO
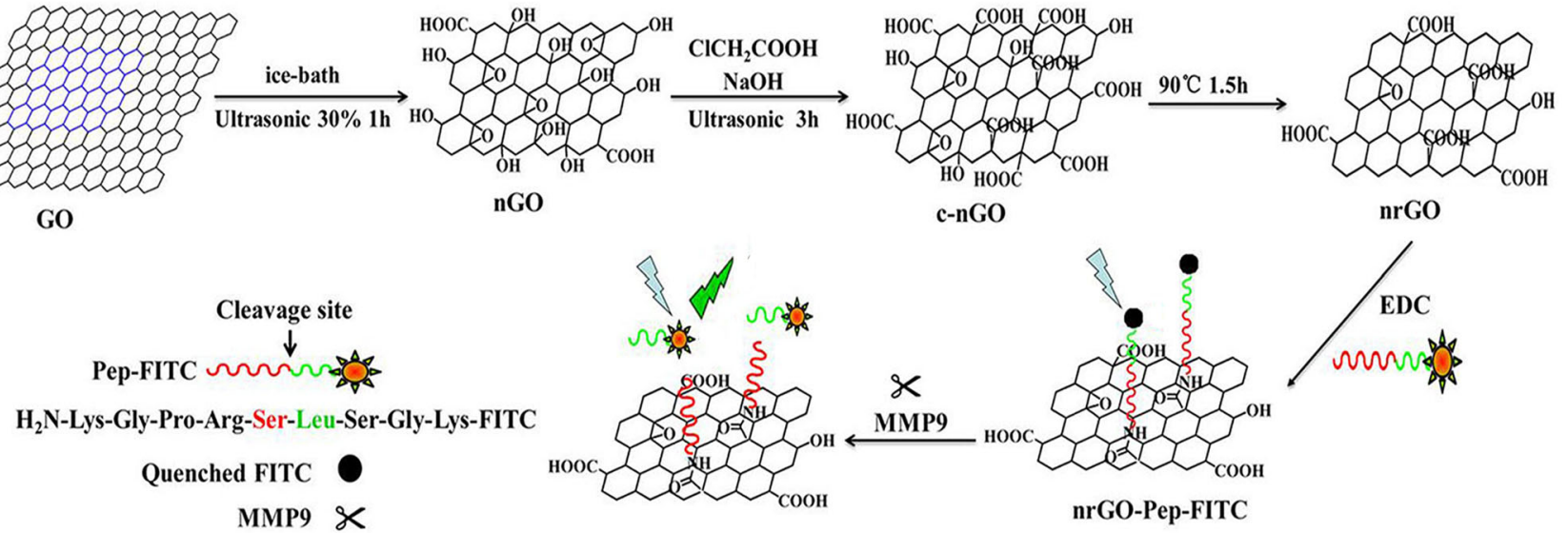

Fig. 1 Strategy of nrGO-Pep-FITC probe for MMP9 detection. Blue arrows indicate $480 \mathrm{~nm}$ excitation light, green arrows indicate emission fluorescence at $518 \mathrm{~nm}$ 


\section{Characterizations}

Characterizations of materials, including atomic force microscopic imaging (AFM), $\zeta$-potentials and polydispersity index (PdI) analysis, ultraviolet-visible (UV-vis) spectra, fourier transfer infrared (FTIR) spectra, and Raman spectra, have been described in our previous publication (Xi et al. 2016). The fluorescence intensity at $518 \mathrm{~nm}$ of FITC with $480 \mathrm{~nm}$ excitation was measured using Luminescence spectrometer (LS 55; PerkinElmer) for the concentration-dependent cleavage of nrGO-PepFITC by MMP9 or microplate reader (Infinite M200; Tecan) for other experiments.

\section{Preparation of nrGO-Pep-FITC nanoprobe}

Preparation of nrGO-Pep-FITC nanoprobe was performed and stored just as described previously (Song et al. 2013). nrGO was dispersed in borate buffer. The mixture of EDC and $\mathrm{nrGO}$ suspension was sonicated for $0.5 \mathrm{~h}$ and then adjusted to $\mathrm{pH} 8.0$ with $\mathrm{NaOH}$. After the addition of $200 \mu \mathrm{L}$ Pep-FITC $(500 \mu \mathrm{M})$, the mixture was stirred for $3 \mathrm{~h}$ in dark at room temperature, and then purified by repeatedly rinsing and centrifuging with $30 \mathrm{kDa}$ ultracentrifuge tube, followed by characterization using UV-Vis.

\section{Stability of nrGO-Pep-FITC nanoprobe}

Effects of BSA and surfactant on nrGO-Pep-FITC were evaluated just as described previously (Song et al. 2013).

\section{Detection of MMP9 with nrGO-Pep-FITC nanoprobe}

Detection of MMP9 with nrGO-Pep-FITC nanoprobe was performed just as described in our previous publication (Xi et al. 2016). After incubation of the activated MMP9 with nrGO-Pep-FITC for $2 \mathrm{~h}$ at $37^{\circ} \mathrm{C}$, fluorescence spectrum of the mixing solution was recorded at room temperature with $480 \mathrm{~nm}$ excitation.

\section{Results and discussion}

\section{Synthesis and characterization of nrGO}

To enhance the level of carboxyl of nrGO for bioapplication, we optimized synthesis conditions through two steps (Fig. 1). Step 1: carboxylation of nGO (c-nGO) by the carboxylation reaction between $\mathrm{nGO}, \mathrm{NaOH}$, and $\mathrm{ClCH}_{2-}$ $\mathrm{COOH}$ for $3 \mathrm{~h}$; Step 2: hydrothermally bathing the c-nGO under $90{ }^{\circ} \mathrm{C}$ for $1.5 \mathrm{~h}$ to obtain reduced c-nGO (nrGO).
Atomic force microscopic images showed that the c-nGO materials obtained by the carboxylation reaction for 3 and $4 \mathrm{~h}$ exhibit a relatively narrow size distribution with about $80 \mathrm{~nm}$ of mean diameter, and about $1.2 \mathrm{~nm}$ of mean thickness (Figure S1). To confirm the dispersibility of these c-nGO materials in aqueous solution, we took their pictures and measured their $\zeta$-potentials and polydispersity index (PdI) using dynamic light scattering (DLS). After storage at $4{ }^{\circ} \mathrm{C}$ for 1 month, c-nGO materials in water show no aggregation response and their color remain nearly unchanged (Figure S2a), indicating excellent water-dispersibility and impressive long-term stability. The mean $\zeta$ potential values less than $-44 \mathrm{mV}$ of these c-nGO materials (Figure S2b) verify that the edges or/and some parts of c-nGO materials are terminated by negatively charged oxygen functional groups such as carboxylic groups, making c-nGO solutions have excellent dispersibility due to electrostatic repulsion mechanism ( $\mathrm{Li}$ et al. 2008). The low PdI values of $0.264,0.259,0.272$, and 0.260 for c-nGO materials obtained by the carboxylation reaction for $1,2,3$, and $4 \mathrm{~h}$, respectively (Figure S2c), further demonstrate their great dispersibility (Bihari et al. 2008).

As shown in Figure S3a, basically superposing UV-Vis spectra of all c-nGO materials demonstrate that the carboxylation time does not affect the UV-Vis absorption spectra of c-nGO materials. FTIR spectrometer was used to characterize chemical groups in the surface of c-nGO materials, and their FTIR spectra have strong absorption peak at $\sim 1600 \mathrm{~cm}^{-1}$ (Figure S3b), demonstrating that these c-nGO materials possess abundant carboxyl groups (COO-). The D band at $1350 \mathrm{~cm}^{-1}$ of Raman spectra is believed to be the characteristic of the defect structure of carbon and the $\mathrm{G}$ band at $1600 \mathrm{~cm}^{-1}$ should be the characteristic of $\mathrm{C} \mathrm{sp}{ }^{2}$ in-plane vibration $\left(\mathrm{E}_{2 \mathrm{~g}}\right.$ mode) (Ferrari et al. 2006). Obviously, the c-nGO obtained by the carboxylation reaction of $\mathrm{nGO}$ for $3 \mathrm{~h}$ shows the highest intensity ratio $\left(I_{\mathrm{D}} / I_{\mathrm{G}}\right)(0.944)$ (Figure $S 3 \mathrm{c}$ ), indicating the highest defect structure of carbon, which may be due to the highest level of chemical interaction between carboxyl groups and GO. So, we chose the c-nGO material obtained by carboxylation reaction of $\mathrm{nGO}$ for $3 \mathrm{~h}$ for following test.

In reality, we hydrothermally bathed c-nGO solution under $90{ }^{\circ} \mathrm{C}$ for 1.5 and $2 \mathrm{~h}$, respectively, to obtain the reduced c-nGO (nrGO [1.5 h] and nrGO [2 h]). Although the $\mathrm{nrGO}[2 \mathrm{~h}]$ exhibits a red-shift of the UV-Vis spectra absorption peak from $237 \mathrm{~nm}$ (nrGO [1.5 h]) to $241 \mathrm{~nm}$ and a higher degree of reduction (Figure S4a), it does not exhibit more excellent fluorescence quenching efficiency over nrGO [1.5 h] (Figure S4b). Therefore, we hydrothermally bathed c-nGO solution under $90{ }^{\circ} \mathrm{C}$ for $1.5 \mathrm{~h}$ to fabricate nrGO.

Figure 2a shows the UV-Vis spectra and digital photo (inset) of nGO, c-nGO, and nrGO. Similar to our previous 
findings (Chen et al. 2014), c-nGO exhibits higher absorbance at long wavelength $(>300 \mathrm{~nm})$ than nGO. nrGO exhibits stronger absorption ability in a broad range from 200 to $900 \mathrm{~nm}$ over c-nGO, which is beneficial to the quenching of most of fluorochromes. Compared to c-nGO, nrGO exhibits $\sim 8.98$-fold increment at $808 \mathrm{~nm}$. Moreover, disappeared shoulder peak $(300 \mathrm{~nm})$ and $\sim 237 \mathrm{~nm}$ absorption peak $(225.5 \mathrm{~nm})$ of $\mathrm{nrGO}$ indicate the effect of deoxygenation and partial restoration of the $\pi$-conjugation network of carbon structure. The color changes of nGO, c-nGO, and nrGO solutions from brown to dark brown (Fig. 2a inset) also verify the reduction of c-nGO. Moreover, nrGO remains stable in water after being stored at $4{ }^{\circ} \mathrm{C}$ for 1 month, which lays a better foundation for subsequent application.

Figure $2 b$ shows the FTIR spectra of nrGO. The nGO characteristic bands present at $\sim 1060 \mathrm{~cm}^{-1}(\mathrm{C}-\mathrm{O})$, $\sim 1380 \mathrm{~cm}^{-1} \quad(\mathrm{C}-\mathrm{OH}), \sim 1620 \mathrm{~cm}^{-1} \quad(\mathrm{C}=\mathrm{C})$, $\sim 1720 \mathrm{~cm}^{-1}(\mathrm{C}=\mathrm{O})$, and $\sim 3400 \mathrm{~cm}^{-1}(\mathrm{OH})$, respectively. After water-bathing treatment, the FTIR spectra of both nrGO and c-nGO have strong absorption peak at $\sim 1600 \mathrm{~cm}^{-1}$ (COO-), demonstrating that a large number of carboxyl groups produced after carboxyl reaction does not disappear after bathing reduction. Raman analysis was also provided to estimate the reduction of c-nGO (Fig. 2c). The intensity ratio $\left(I_{\mathrm{D}} / I_{\mathrm{G}}\right)$ of $\mathrm{D}$ band to $\mathrm{G}$ band is $\sim 0.815$ for nGO, 0.944 for $\mathrm{c}-\mathrm{nGO}$, and 0.856 for nrGO. The much higher $I_{\mathrm{D}} / I_{\mathrm{G}}$ value of c-nGO than that of nGO indicates the great damage of the regularity of nGO by carboxyl reaction, while the lower $I_{\mathrm{D}} / I_{\mathrm{G}}$ value of nrGO than that of c-nGO suggests that the green reduction reaction recovers the aromatic structures.

Atomic force microscopic image shows that $\mathrm{nrGO}$ presents about $80 \mathrm{~nm}$ in sheet diameter, and $\sim 1.2 \mathrm{~nm}$ of sheet thickness (Fig. 3a). As shown in Fig. 3b, c, nrGO remains $\sim-41.7 \mathrm{mV}$ of $\zeta$-potentials and $\sim 0.16$ of PdI, demonstrating the great dispersibility of $\mathrm{nrGO}$ in water.

\section{Preparation and optimization of nrGO-Pep-FITC nanoprobe}

To get the highest fluorescence quenching efficiency of nrGO for the Pep-FITC and save the usage of nrGO, the concentration of nrGO was optimized. Different concentrations of nrGO solutions were incubated with Pep-FITC in borate buffer for $20 \mathrm{~min}$, and then their fluorescence intensities were recorded using microplate reader. In addition, different concentrations of c-nGO were incubated with Pep-FITC for comparison. As shown in Fig. 4, fluorescence intensity is gradually weakened along with the increasing concentrations of nrGO and c-nGO. nrGO has much higher fluorescence quenching ability than c-nGO at the concentration range $0-2 \mu \mathrm{g} / \mathrm{mL}$, and $4 \mu \mathrm{g} / \mathrm{mL}$ nrGO and $10 \mu \mathrm{g} / \mathrm{mL}$ c-nGO exhibit about $95 \%$ of fluorescence quenching efficiency to the Pep-FITC, indicating the stronger quenching ability of nrGO over c-nGO due to the strong absorption ability of nrGO (Xi et al. 2016). Therefore, we chose $4 \mu \mathrm{g} / \mathrm{mL}$ nrGO and $1 \mu \mathrm{M}$ Pep-FITC for the following nanoprobe preparation.

We measured absorption spectra of materials to assess the linking of Pep-FITC to nrGO. Covalent nrGO-PepFITC nanoprobe was fabricated by chemical reaction between $20 \mu \mathrm{g} / \mathrm{mL}$ nrGO and $5 \mu \mathrm{M}$ Pep-FITC under the participation of EDC, followed by repeated filtering to remove free Pep-FITC in solution, while non-covalent nrGO/Pep-FITC nanoprobe was prepared by simple mixing of $20 \mu \mathrm{g} / \mathrm{mL}$ nrGO with $5 \mu \mathrm{M}$ Pep-FITC without filtering. As shown in Fig. 5, compared to nrGO, nrGO/Pep-FITC exhibits the characteristic absorption peaks $(480 \mathrm{~nm})$ of Pep-FITC superimposing on the nrGO absorption curve. The basically superposing absorption spectra of the two nanoprobes suggest that a large number of Pep-FITC is connected to nrGO.

BSA was employed to investigate the stability of the two probes. We used microplate reader to measure the enhanced fluorescence intensity $\Delta F$ (Song et al. 2013). (a)

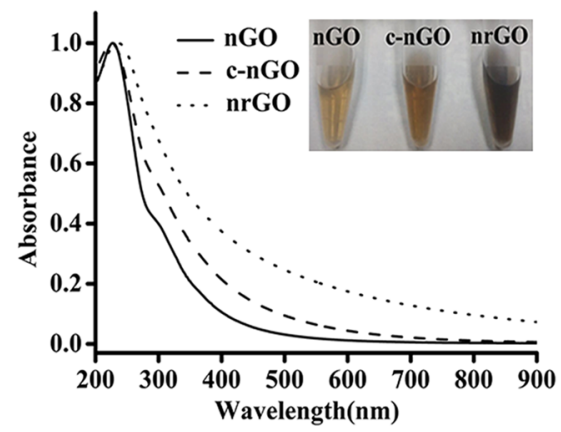

(b)

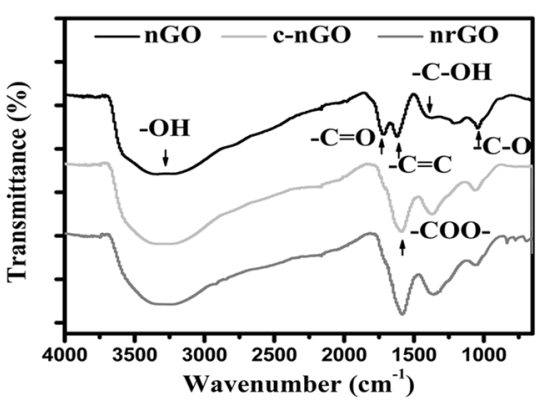

(c)

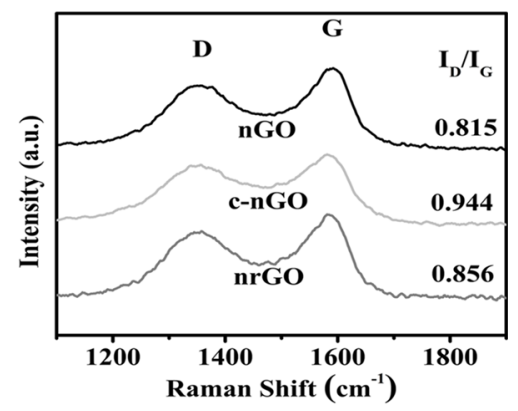

Fig. 2 Characterizations of nano-materials. a UV-Vis absorption spectra of nGO, c-nGO, and nrGO. Inset shows their photograph after storage at $4{ }^{\circ} \mathrm{C}$ for 1 month. b and c FTIR spectra (b) and Raman spectra (c) of nGO, c-nGO, and nrGO 
(a)

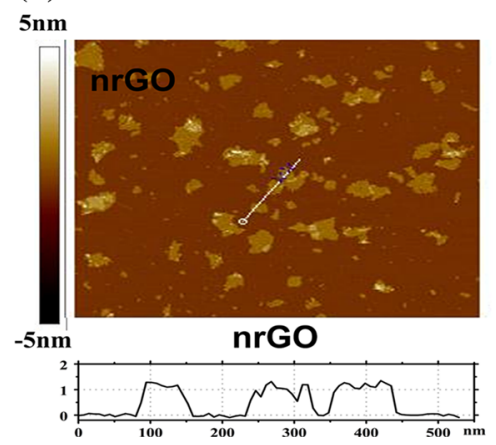

(b)

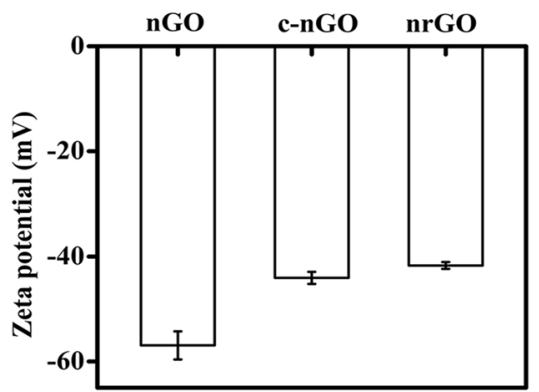

(c)

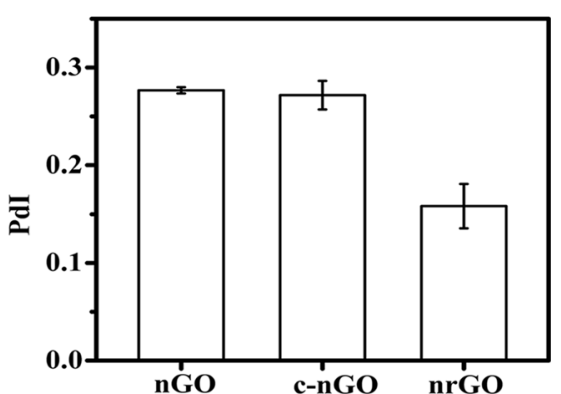

Fig. 3 a AFM image of nrGO. Inset indicates AFM thickness analysis of nrGO. b Zeta potentials of nGO, c-nGO, and nrGO after storage at $4{ }^{\circ} \mathrm{C}$ for 1 month. c Polydispersity index (PdI) of nGO, c-nGO, and nrGO after storage at $4{ }^{\circ} \mathrm{C}$ for 1 month

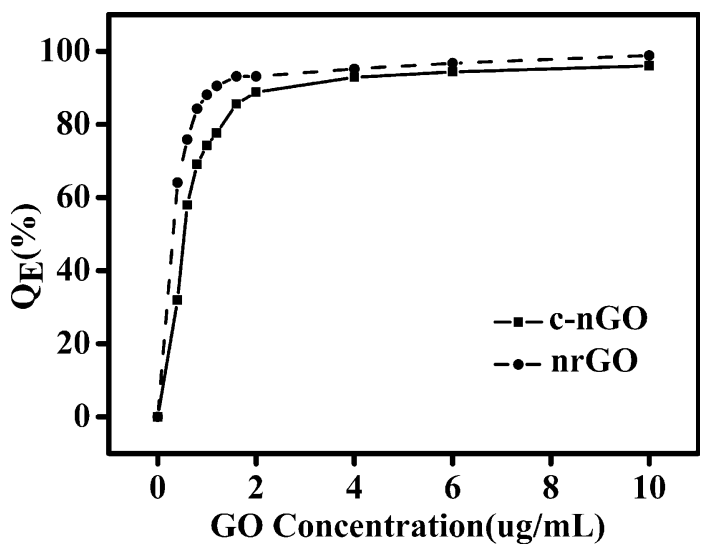

Fig. 4 Quenching efficiencies of nano-materials. Dose-dependent quenching efficiencies $\left(\mathrm{Q}_{\mathrm{E}}\right)$ of c-nGO and nrGO on Pep-FITC $(1 \mu \mathrm{M})$ in borate buffer

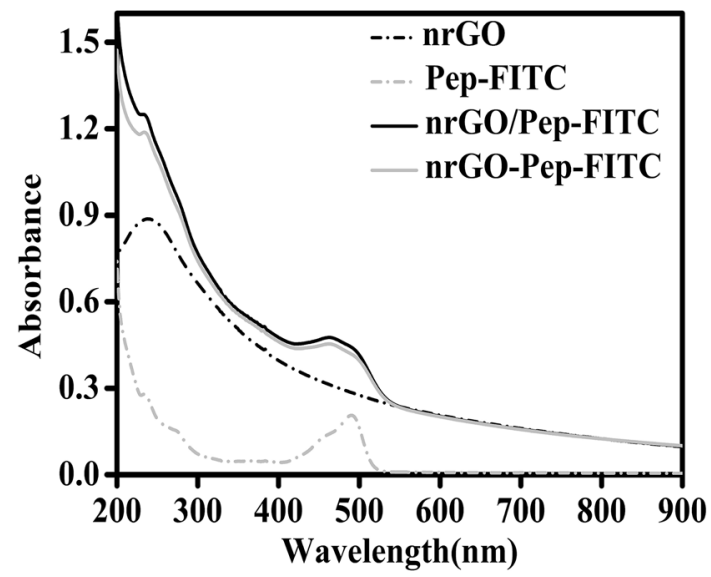

Fig. 5 UV-Vis absorption spectra of nrGO $(20 \mu \mathrm{g} / \mathrm{mL})$, Pep-FITC $(5 \mu \mathrm{M})$, nrGO/Pep-FITC $(5 \mu \mathrm{M})$, and nrGO-Pep-FITC $(5 \mu \mathrm{M})$

nrGO/Pep-FITC exhibited a distinctly increased fluorescence intensity, while nrGO-Pep-FITC nanoprobe only exhibits a very slight increase even when $500 \mu \mathrm{g} / \mathrm{mL}$ of BSA is added (Fig. 6a), which is due to its covalent binding. The slight increase in fluorescence intensity of
nrGO-Pep-FITC nanoprobe in the presence of BSA is likely due to the displacement of small partial of Pep-FITC noncovalently binding to nrGO by BSA. The stability of nrGO-Pep-FITC nanoprobe to some common surfactants was assessed. As shown in Fig. 6b, after adding 0.1\% Triton 100 or Tween 20, nrGO/Pep-FITC nanoprobe shows a significant fluorescence enhancement, while nrGO-PepFITC nanoprobe has much less response. These results clearly demonstrate the excellent stability of nrGO-PepFITC nanoprobe than nrGO/Pep-FITC nanoprobe against other environmental molecules/conditions.

\section{Detection of MMP9 using nrGO-Pep-FITC nanoprobe}

We next tested its ability to detect MMP9 activity. Different concentrations of activated MMP9 were incubated with nrGO-Pep-FITC $(1 \mu \mathrm{M})$ for $2 \mathrm{~h}\left(37^{\circ} \mathrm{C}\right.$ in borate buffer), followed by recording the fluorescence signal of each sample using Luminescence spectrometer with $480 \mathrm{~nm}$ excitation. As shown in Fig. 7, the fluorescence intensity of nrGO-Pep-FITC increases with MMP9 concentration range from 0 to $0.25 \mathrm{nM}$, and a good linear

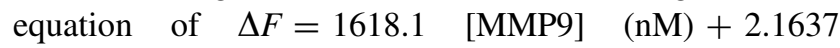
$(r=0.9961)$ is obtained in the range $0.01-0.06 \mathrm{nM}$ MMP9. The detection limit is $0.83 \mathrm{pM}$, about 180 -fold lower than the detection limit of previously reported MMP9 FRET nanoprobes (Nguyen et al. 2017). In addition, the $\Delta F$ is also linearly correlated with MMP9 concentration in the range $0.06-0.15 \mathrm{nM}$.

The two linear detection ranges of nrGO-Pep-FITC for MMP9 may be due to two binding manners of Pep-FITC to nrGO as shown in Figure S5. One manner is covalent binding through the formation of amide bond between the $\mathrm{NH}_{2}$ groups of peptide and the $-\mathrm{COOH}$ groups of nrGO. Because of complete exposure, the cleavage site of PepFITC can be easily cleaved by MMP9, which leads to the ultrasensitive linear detection range of nrGO-Pep-FITC 
Fig. 6 Stability of nrGO-PepFITC. Enhanced fluorescence intensity $(\Delta F)$ of nrGO/PepFITC and nrGO-Pep-FITC in the presence of different concentrations of a BSA and b $0.1 \%$ Triton 100 or Tween 20 (a)

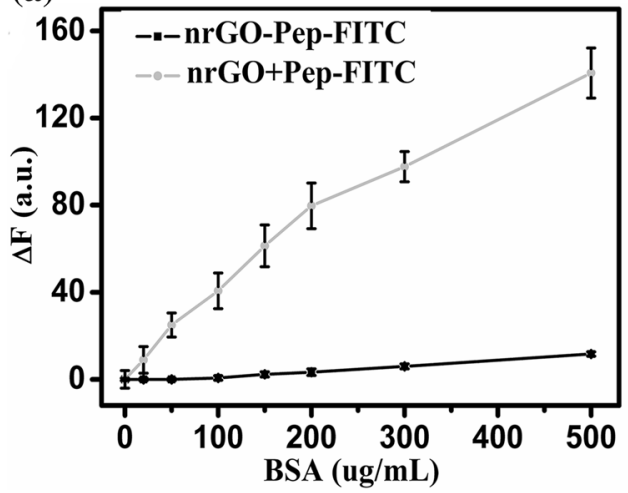

(b)

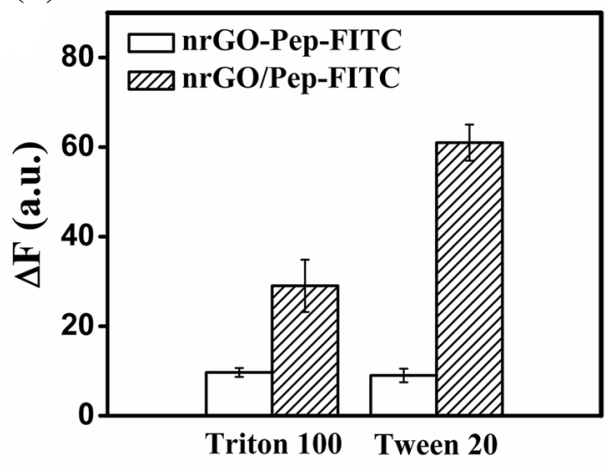

(a)

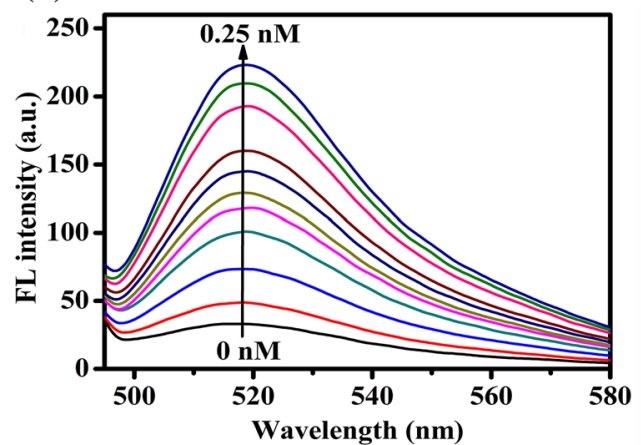

Fig. 7 Concentration-dependent cleavage of nrGO-Pep-FITC by MMP9. a Fluorescence spectra of nrGO-Pep-FITC in the presence of various concentrations of MMP9 $(0,0.01,0.02,0.04,0.05,0.06$, $0.08,0.1,0.15,0.2$, and $0.25 \mathrm{nM})$. b Plot of $\Delta F$ versus the concentration of MMP9 from 0 to $0.25 \mathrm{nM}$. Insets at the bottom

from 0.01 to $0.06 \mathrm{nM}$ for MMP9. Another manner is covalent and non-covalent ( $\pi-\pi$ stacking and electrostatic interaction) bindings of Pep-FITC to nrGO, which increase the steric hindrance of the cleavage site of Pep-FITC, leading to another linear detection range of nrGO-PepFITC from 0.06 to $0.15 \mathrm{nM}$ for MMP9.

The ultrasensitive detection ability of nrGO-Pep-FITC nanoprobe for MMP9 is due to the higher quenching ability of nrGO to visible light. The $\sim 8$.98-fold increment at $808 \mathrm{~nm}$ (Fig. 2a) potently enhances the fluorescence quenching efficiency of nrGO. Although increasing the reduction degree of nrGO can further enhance the absorption of nrGO to visible light (Fan et al. 2008), it not only removes more oxygen-containing groups but also results in aggregation and precipitating of $\mathrm{nrGO}$, which is not beneficial to the covalent linking between the $-\mathrm{COOH}$ groups of nrGO and the $-\mathrm{NH}_{2}$ groups of Pep-FITC. Furthermore, we recently found that the fluorescence quenching ability of nrGO couldn't be further improved obviously with the increasing reduction degree of GO when the absorption peak of nrGO red-shifted up to $236.5 \mathrm{~nm}$ (Xi et al. 2016). We thus chose nrGO with $236.5 \mathrm{~nm}$ of (b)

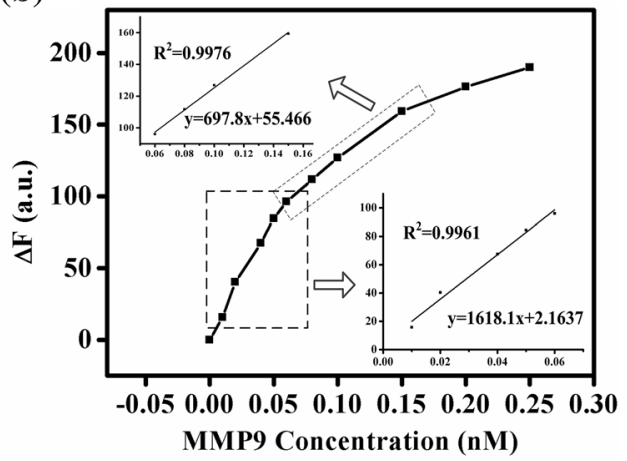

right and the top left indicate the linear regression of $\Delta F$ versus the concentration of MMP9. Linear detection ranges are indicated by dotted rectangle. $\Delta F$ is the enhanced fluorescence intensity of nrGOPep-FITC in the presence of MMP9. $\lambda$ ex/em $=480 / 518 \mathrm{~nm}$

absorption peak to synthesize the covalent nrGO-Pep-FITC probe.

It was reported that MMP9 concentration in the synovial fluid of patients with osteoarthritis was 3.47-9.77 pM (Kim et al. 2010) and MMP9 concentration in the cerebrospinal fluid of patients after epileptic seizures was $50-102 \mathrm{pM}(\mathrm{Li}$ et al. 2013). MMP9 below $150 \mathrm{pM}$ cannot be analyzed by GO-based FRET sensor developed previously (Nguyen et al. 2017). The ultrasensitive detection ability of nrGOPep-FITC developed here makes it possible to exactly detect MMP9 as a biological marker for early disease detection.

\section{Specificity of nrGO-Pep-FITC nanoprobe toward MMP9}

To test the specificity of nrGO-Pep-FITC nanoprobe for MMP9, various potentially interfering substances, such as inorganic salts $\left(\mathrm{ZnCl}_{2}, \mathrm{MgCl}_{2}\right.$, and $\left.\mathrm{KCl}\right)$, glutamine, glycine, glucose, human serum albumin (HSA), BSA, and MMP2, were examined under the same conditions by detecting the fluorescence intensities of mixing solution 


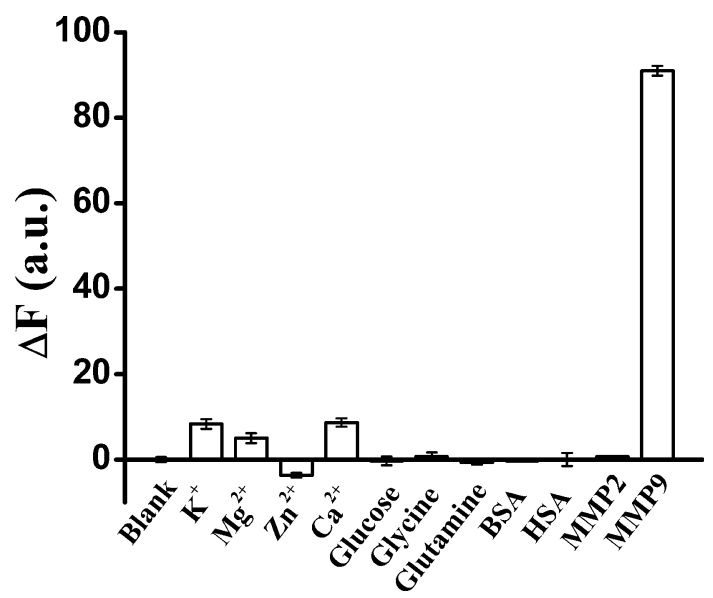

Fig. 8 Fluorescence responses of nrGO-Pep-FITC $(1 \mu \mathrm{M})$ to various substances: $\mathrm{KCl}(15 \mathrm{mM}), \mathrm{MgCl}_{2}(0.25 \mathrm{mM}), \mathrm{ZnCl}_{2}(0.25 \mathrm{mM})$, $\mathrm{CaCl}_{2}(0.25 \mathrm{mM})$, glucose $(1 \mathrm{mM})$, glycine $(0.1 \mathrm{mM})$, glutamine $(0.1 \mathrm{mM})$, BSA $(10 \mathrm{nM})$, HSA $(10 \mathrm{nM})$, MMP9 $(0.25 \mathrm{nM})$, and MMP2 $(0.25 \mathrm{nM}) . \Delta F$ is the enhanced fluorescence intensity of nrGO-Pep-FITC in the presence of a substance

using microplate reader. As shown in Fig. 8, nrGO-PepFITC nanoprobe exhibits the strongest fluorescence response to MMP9 even with a concentration of $0.25 \mathrm{nM}$. nrGO-Pep-FITC nanoprobe does not response obviously to $\mathrm{ZnCl}_{2}, \mathrm{MgCl}_{2}, \mathrm{KCl}$, glutamine, glycine, glucose, $\mathrm{HSA}$, and BSA even with micromole-level concentrations. Moreover, other matrix metalloproteinases, such as MMP2, at the same concentration as that of MMP9 used above $(0.25 \mathrm{nM})$, have little effect on the fluorescence response of nrGO-Pep-FITC nanoprobe. These data clearly demonstrate the specific detection ability of nrGO-Pep-FITC nanoprobe for MMP9.

Blood and urine, the commonly used clinical samples for MMP9 detection, contain amino acid, saccharide, saline, and other MMPs. The specificity of nrGO-Pep-FITC nanoprobe for MMP9 detection has great significance for clinical diagnosis. We are currently optimizing the synthesis of nrGO-Pep-FITC nanoprobe to further enhance its stability and suitability in blood and urine, and this nanoprobe will be applied to quantitatively analyze clinical blood and/or urine samples from patients.

\section{Conclusions}

In summary, we developed a stable and covalent nrGOPep-FITC nanoprobe for ultrasensitive detection of MMP9. Compared to non-covalent nanoprobe, nrGO-Pep-FITC nanoprobe is more stable to external environment interferences. Moreover, this nanoprobe exhibits $0.83 \mathrm{pM}$ of detection limit for MMP9. The nrGO-Pep-FITC developed here not only is the first nrGO-based probe for analyzing
MMP9 but also has the lowest detection limit for MMP9 up to now, which makes it possible to use MMP9 as a reliable candidate for diagnostic biomarker of disease in early stage.

Acknowledgements This work was supported by the National Natural Science Foundation of China (Grants 81572184 and 81471699) and the Natural Science Foundation of Guangdong Province (Grant 2014A030313378) as well as the Fundamental Research Funds for the First Clinical Medicine College of Jinan University (Grant 2015108).

Open Access This article is distributed under the terms of the Creative Commons Attribution 4.0 International License (http:// creativecommons.org/licenses/by/4.0/), which permits unrestricted use, distribution, and reproduction in any medium, provided you give appropriate credit to the original author(s) and the source, provide a link to the Creative Commons license, and indicate if changes were made.

\section{References}

Akers WJ, Xu B, Lee H, Sudlow GP, Fields GB, Achilefu S, Edwards WB (2012) Detection of MMP-2 and MMP-9 activity in vivo with a triple-helical peptide optical probe. Bioconjug Chem 23:656-663. doi:10.1021/bc300027y

Berk BC, Fujiwara K, Lehoux S (2007) ECM remodeling in hypertensive heart disease. J Clin Investig 117:568-575. doi:10.1172/JCI31044

Bihari P, Vippola M, Schultes S, Praetner M, Khandoga AG, Reichel CA, Coester C, Tuomi T, Rehberg M, Krombach F (2008) Optimized dispersion of nanoparticles for biological in vitro and in vivo studies. Part Fibre Toxicol 5:14. doi:10.1186/1743-89775-14

Burrage PS, Mix KS, Brinckerhoff CE (2006) Matrix metalloproteinases: role in arthritis. Front Biosci 11:529-543

Celentano DC, Frishman WH (1997) Matrix metalloproteinases and coronary artery disease: a novel therapeutic target. J Clin Pharmacol 37:991-1000

Chen J, Wang X, Chen T (2014) Facile and green reduction of covalently PEGylated nanographene oxide via a 'water-only' $r$ oute for high-efficiency photothermal therapy. Nanoscale Res Lett 9:86. doi:10.1186/1556-276X-9-86

Eissa S, Ali-Labib R, Swellam M, Bassiony M, Tash F, El-Zayat TM (2007) Noninvasive diagnosis of bladder cancer by detection of matrix metalloproteinases (MMP-2 and MMP-9) and their inhibitor (TIMP-2) in urine. Eur Urol 52:1388-1396. doi:10. 1016/j.eururo.2007.04.006

Fan X, Peng W, Li Y, Li X, Wang S, Zhang G, Zhang F (2008) Deoxygenation of exfoliated graphite oxide under alkaline conditions: a green route to graphene preparation. Adv Mater 20:4490-4493. doi:10.1002/adma.200801306

Feng D, Zhang Y, Feng T, Shi W, Li X, Ma H (2011) A graphene oxide-peptide fluorescence sensor tailor-made for simple and sensitive detection of matrix metalloproteinase 2. Chem Commun 47:10680-10682. doi:10.1039/c1cc13975d

Fernández CA, Yan L, Louis G, Yang J, Kutok JL, Moses MA (2005) The matrix metalloproteinase-9/neutrophil gelatinase-associated lipocalin complex plays a role in breast tumor growth and is present in the urine of breast cancer patients. Clin Cancer Res 11:5390-5395. doi:10.1158/1078-0432.CCR-04-2391 
Ferrari AC, Meyer JC, Scardaci V, Casiraghi C, Lazzeri M, Mauri F, Piscanec S, Jiang D, Novoselov KS, Roth S, Geim AK (2006) Raman spectrum of graphene and graphene layers. Phys Rev Lett 97:187401. doi:10.1103/PhysRevLett.97.187401

Fudala R, Rich R, Mukerjee A, Ranjan A, Vishwanatha J, Kurdowska A, Gryczynski Z, Borejdo J, Gryczynski I (2014) Fluorescence detection of MMP-9. II. Ratiometric FRET-based sensing with dually labeled specific peptide. Curr Pharm Biotechnol 14:1134-1138. doi:10.2174/138920101413140605111109

Fujimura M, Gasche Y, Morita-Fujimura Y, Massengale J, Kawase M, Chan PH (1999) Early appearance of activated matrix metalloproteinase- 9 and blood-brain barrier disruption in mice after focal cerebral ischemia and reperfusion. Brain Res 842:92-100

Giebel SJ, Menicucci G, McGuire PG, Das A (2005) Matrix metalloproteinases in early diabetic retinopathy and their role in alteration of the blood-retinal barrier. Lab Investig 85:597-607. doi:10.1038/labinvest.3700251

Hurst NG, Stocken DD, Wilson S, Keh C, Wakelam MJ, Ismail T (2007) Elevated serum matrix metalloproteinase 9 (MMP-9) concentration predicts the presence of colorectal neoplasia in symptomatic patients. Br J Cancer 97:971-977. doi:10.1038/sj. bjc. 6603958

Kim KS, Choi HM, Lee YA, Choi IA, Lee SH, Hong SJ, Yang HI, Yoo MC (2010) Expression levels and association of gelatinases MMP-2 and MMP-9 and collagenases MMP-1 and MMP-13 with VEGF in synovial fluid of patients with arthritis. Rheumatol Int 31:543-547. doi:10.1007/s00296-010-1592-1

Kuyvenhoven JP, Molenaar IQ, Verspaget HW, Veldman MG, Palareti G, Legnani C, Moolenburgh SE, Terpstra OT, Lamers CB, van Hoek B, Porte RJ (2004) Plasma MMP-2 and MMP-9 and their inhibitors TIMP-1 and TIMP-2 during human orthotopic liver transplantation. The effect of aprotinin and the relation to ischemia/reperfusion injury. Thromb Haemost 91:506-513. doi:10.1160/TH03-05-0272

Li D, Muller MB, Gilje S, Kaner RB, Wallace GG (2008) Processable aqueous dispersions of graphene nanosheets. Nat Nanotechnol 3:101-105. doi:10.1038/nnano.2007.451

Li J, Lu CH, Yao QH, Zhang XL, Liu JJ, Yang HH, Chen GN (2011) A graphene oxide platform for energy transfer-based detection of protease activity. Biosens Bioelectron 26:3894-3899. doi:10. 1016/j.bios.2011.03.003

Li YJ, Wang ZH, Zhang B, Zhe X, Wang MJ, Shi ST, Bai J, Lin T, Guo CJ, Zhang SJ, Kong XL, Zuo X, Zhao H (2013) Disruption of the blood-brain barrier after generalized tonic-clonic seizures correlates with cerebrospinal fluid MMP-9 levels. J Neuroinflamm 10:80. doi:10.1186/1742-2094-10-80

Li X, Deng D, Xue J, Qu L, Achilefu S, Gu Y (2014) Quantum dots based molecular beacons for in vitro and in vivo detection of MMP-2 on tumor. Biosens Bioelectron 61:512-518. doi:10. 1016/j.bios.2014.05.035

Li SY, Liu LH, Cheng H, Li B, Qiu WX, Zhang XZ (2015) A dualFRET-based fluorescence probe for the sequential detection of MMP-2 and caspase-3. Chem Commun 51:14520-14523. doi: $10.1039 / \mathrm{c} 5 \mathrm{cc} 04962 \mathrm{~h}$
Martin MD, Matrisian LM (2007) The other side of MMPs: protective roles in tumor progression. Cancer Metastasis Rev 26:717-724. doi:10.1007/s10555-007-9089-4

Mroczko B, Kozlowski M, Groblewska M, Lukaszewicz M, Niklinski J, Jelski W, Laudanski J, Chyczewski L, Szmitkowski M (2008) The diagnostic value of the measurement of matrix metalloproteinase 9 (MMP-9), squamous cell cancer antigen (SCC) and carcinoembryonic antigen (CEA) in the sera of esophageal cancer patients. Clin Chim Acta 389:61-66. doi:10.1016/j.cca. 2007.11.023

Nguyen PD, Cong VT, Baek C, Min J (2017) Fabrication of peptide stabilized fluorescent gold nanocluster/graphene oxide nanocomplex and its application in turn-on detection of metalloproteinase-9. Biosens Bioelectron 89:666-672. doi:10.1016/j.bios. 2015.12.031

Riedel F, Gotte K, Schwalb J, Bergler W, Hormann K (2000) Expression of $92-\mathrm{kDa}$ type IV collagenase correlates with angiogenic markers and poor survival in head and neck squamous cell carcinoma. Int J Oncol 17:1099-1106

Rosenberg GA, Estrada EY, Dencoff JE (1998) Matrix metalloproteinases and TIMPs are associated with blood-brain barrier opening after reperfusion in rat brain. Stroke 29:2189-2195

Shpitzer T, Hamzany Y, Bahar G, Feinmesser R, Savulescu D, Borovoi I, Gavish M, Nagler RM (2009) Salivary analysis of oral cancer biomarkers. Br J Cancer 101:1194-1198. doi:10.1038/sj. bjc. 6605290

Song E, Cheng D, Song Y, Jiang M, Yu J, Wang Y (2013) A graphene oxide-based FRET sensor for rapid and sensitive detection of matrix metalloproteinase 2 in human serum sample. Biosens Bioelectron 47:445-450. doi:10.1016/j.bios.2013.03.030

Sternlicht MD, Werb Z (2001) How matrix metalloproteinases regulate cell behavior. Annu Rev Cell Dev Biol 17:463-516. doi:10.1146/annurev.cellbio.17.1.463

Sun X, Liu Z, Welsher K, Robinson JT, Goodwin A, Zaric S, Dai H (2008) Nano-graphene oxide for cellular imaging and drug delivery. Nano Res 1:203-212. doi:10.1007/s12274-008-8021827

Wang Z, Li X, Feng D, Li L, Shi W, Ma H (2014) Poly (mphenylenediamine)-based fluorescent nanoprobe for ultrasensitive detection of matrix metalloproteinase 2. Anal Chem 86:7719-7725. doi:10.1021/ac5016563

Xi G, Wang X, Chen T (2016) A reduced graphene oxide-based fluorescence resonance energy transfer sensor for highly sensitive detection of matrix metalloproteinase 2. Int $\mathrm{J}$ Nanomed 11:1537-1547. doi:10.2147/IJN.S102517

Yu Q, Stamenkovic I (2000) Cell surface-localized matrix metalloproteinase-9 proteolytically activates TGF- $\beta$ and promotes tumor invasion and angiogenesis. Genes Dev 14:163-176

\section{Publisher's Note}

Springer Nature remains neutral with regard to jurisdictional claims in published maps and institutional affiliations. 